\title{
Numbers, Geometry, and Mathematical Axioms: The Problem of Metaphysics in the Critique of Pure Reason
}

\author{
Kazuhiko Yamamoto
}

Megumi Institute of Ethics and Philosophy, Japan

\begin{abstract}
So far, we have clarified that 1) "the representation I think" - the transcendental unity of selfconsciousness - is homogeneous with pure apperception which signifies the thoroughgoing identity of oneself in all possible representations, grounding empirical consciousness a priori: 2) "the representation I think" which can accompany all others, is to cognize through categories whatever objects may come before our senses. Thus we comprehend that a human, as "the representation I think" senses, intuits and cognizes all appearances themselves ins virtue of filled space-elapsing time or nullity in space-time through empirical intuition and synthesis. Our transcendental analytic indicates that a being of all beings signifies space-time itself, i.e., quantum. Kant's metaphysics, which states that the members of the division exclude each other and yet are connected in one sphere, so in the latter case the parts are represented as ones to which existence (as substances) pertains to each exclusively of the others, and which are yet connected in one whole, led us to think that "the members of the division" signifies categories, through which it would become possible for us to cognize any object as far as laws of their combination are concerned. The discourse would potentially lead us to an alternative view on the universe and causality. We feel that our transcendental analytic might give us an inkling for the solution of conundrums in mathematics and physics.
\end{abstract}

Keywords: Numbers, Geometry, Mathematical Axioms, Metaphysics

\section{INTRODUCTION}

Kant's negative remarks toward "the mathematical investigators of nature" (B56 ${ }^{1}$ ) and "some metaphysicians of nature" (A40) can be thought to reveal his basic position toward mathematics and metaphysics: 1) Kant does not "assume two eternal and infinite self-subsisting non-entities (space and time), which exists (yet without there being anything real)" (B56); 2) he does not "hold space and time to be relations of appearances (next to or successive to one another) that are abstracted from experience" (A40-B57). We think that this position clearly shows us how erroneously Kant comprehends space and time (YAMAMOTO 2017b: 72-81). Kant, who believes that "two eternal and infinite self-subsisting non-entities" have nothing to do with anything real, thinks that "relations of appearances (next to or successive to one another) that are abstracted from experience" (A40-B57) have little relevance because they are "confusedly represented in this abstraction" (B57). Kant seems to think that the abstraction that can "occur without the restrictions that nature has attached to them" (B57) is relevant. He says, "There is no other way than through concepts or through intuitions, both of which, however, are given, as such, either a priori or a posteriori. The latter, namely empirical concepts, together with that on which they are grounded, empirical intuition, cannot yield any synthetic proposition except one that is also merely empirical, i.e., a proposition of experience; thus it can never contain necessity and absolute universality of the sort that is nevertheless characteristic of all propositions of geometry" (A47). We think that this is wrong. We have to think that since the transcendental unity of apperception, which comprises pure intuition, an a priori intuition and pure concept of the understanding, has arisen as "intuitions themselves" (B160) and "perceptions themselves" (B219), it can make a "proposition of experience" as a "synthetic proposition" which can "contain necessity and absolute universality" (A47) - nullity in space-time - space-time itself (YAMAMOTO 2016: 87-100, YAMAMOTO 2017a: 19-37, YAMAMOTO 2017b: 72-81). Why Kant cannot reach space-time itself - nullity in space-time - through empirical intuition and synthesis

1 B56 designates the pagination of the standard German edition of Kant's works, as indicated by means of marginal numbers in Critique of Pure Reason (Kant, Immanuel, Critique of Pure Reason, Cambridge University Press, 1999). All citations are the same. 


\section{Kazuhiko Yamamoto}

can be seen in what he refers to in regard to geometry, which says, "Since the propositions of geometry are cognized synthetically a priori and with apodictic certainty, I ask: Whence do you take such propositions, and on what does our understanding rely in attaining to such absolutely necessary and universally valid truths?" (B64-A47). Here we have to ask Kant, who, do you think, can cognize the propositions of geometry synthetically a priori and with apodictic certainty? It is only humans, who have appeared after geometry had been introduced, as a science, among their consciousness in the ancient Greek world, that can cognize the propositions of geometry. Thus, in regard to the propositions of geometry as a science, we have to take anthropocentric and anthropological factors into consideration (YAMAMOTO 2017a: 19-37). Kant, who acknowledges that it might not have universality, saying, "Although it is synthetic, however, it is still only a singular proposition" (B205), has missed a crucial point, which is clear in his saying, "Insofar as it is only the synthesis of that which is homogeneous (of units) that is at issue here, the synthesis here can take place only in a single way, even though the subsequent use of these numbers is general" (B205). What does this mean? It indicates Kant's way of thinking. No, it is not possible among humans in primordial times that "with three lines, two of which taken together are greater than the third, a triangle can be drawn" (B205). It is not farfetched to assume that, in primordial times, human did not have the "mere function of the productive imagination, which draws the lines greater or smaller, thus allowing them to abut at any arbitrary angle" (B205-A165) "without the restrictions that nature has attached to them" (B57). Moreover, the number 7 is not "possible in only a single way" (A165). Therefore, we have to think that as Kant says, "such propositions must therefore not be called axioms (for otherwise there would be infinitely many of them) but rather numerical formulas" (A165-B206). In this regard, we have already said as follows: When we try to find what empirical intuition means among the standing and lasting Is, we have to know how the standing and lasting Is cognize things "as they appear" (A250), through empirical intuition, in the milieu in which geometry or mathematics, as a science, does not wield overwhelming power on the imagination. We think that if geometry or mathematics, as a science, affects the imagination strongly, it might acclimatize empirical intuition along with its power of abstraction since imagination, as "the faculty for representing an object even without its presence in intuition" (B151), is "the subjective condition" which might "give a corresponding intuition to the concepts of understanding" (B151) (YAMAMOTO 2017a: 19-37): we hypothesize that 1) imagination is the pristine faculty of humans by virtue of "an exercise of spontaneity" (B151), through which it is possible to cognize objects as appearance in filled space-elapsing time, and the disappearance of the objects as appearance in empty space-nullified time, 2) since pure intuition is the empirical intuition from which everything that belongs to sensation is detached, pure intuition affected by the sensation of nullity can be homogeneous with empirical intuition, 3) when humans empirically cognize objects as appearance in filled space-elapsing time, they comprehend it to be space-time which pertains to objects as appearance, 4) filled space-elapsing time - appearance itself is understood to be "all objects" as "mere appearances" (A49) on account of the fact that the imagination is affected by geometry or mathematics in terms of scientific knowledge (YAMAMOTO 2017a: 19-37).

We have to assume that, in primordial times, when humans' imagination was not affected by geometry or mathematics in terms of scientific knowledge, they had the "synthesis of spaces and times, as the essential form of all intuition... which at the same time makes possible the apprehension of the appearance, thus every outer experience, consequently also all cognition of its objects" (B206-A166) in conjunction with experience or possible experience of death. We have already clarified that Kant's "pure concept of the understanding" (A142) is homogeneous with death itself, namely nullity in space-time (YAMAMOTO 2016: 87-100). In view of the fact that "a synthesis of perceptions, which is not itself contained in perception" (B218) is to be combined with experience, i.e., "a cognition that determines an object through perceptions" (B218) or with possible experience, i.e., the possibility of "a cognition that determines an object through" (B218) possible perceptions, it comes out that nullity in space-time, "as conditions of a possible experience" (A139) or "as conditions of the possibility of things in general" (A139), "can be extended to objects in themselves" (A139). In other words, it can be said, in an opposite manner to what Kant refers to (B57-A41), that nullity in space-time can offer ground for the possibility of a priori mathematical cognitions (since it has a true and objectively valid a priori intuition), and can it bring the propositions of experience into necessary accord with those assertions. We think that living things became human beings upon acquiring the "pure concept of the understanding" (A142) in virtue of nullity in space-time, enabling them to cognize the existence of appearances - appearances themselves. The nullity in space-time signifies "the a priori concepts of 
space and time" (B57), which are not "creatures of the imagination, the origin of which must really be sought in experience, out of whose abstracted relations imagination has made something that, to be sure, contains what is general in them, but that cannot occur without the restrictions that nature has attached to them" (B57). Since "nowhere beyond the field of possible experience can there be any synthetic a priori principles" (A248-B305), "all of our cognitions" (A146), which "lie in the entirety of all possible experience" (A146), can be enhanced to "synthetic a priori principles" (B305), i.e., "transcendental truth, which precedes all empirical truth and makes it possible" (A146). When Kant says, "The postulate for cognizing the actuality of things requires perception, thus sensation of which one is conscious - not immediate perception of the object itself the existence of which is to be cognized, but still its connection with some actual perception in accordance with the analogies of experience, which exhibit all real connection in an experience in general" (A225), we entirely agree with him, thinking that, in this context, "all possible experience" (A146) pertain to "some actual perception in accordance with the analogies of experience" (A225). Since the category, drawn from "all of our cognitions" (A146) which "lie in the entirety of all possible experience" (A146), is to signify "every universal proposition, even if it is taken from experience (by deduction)" (A300), it "can serve as the major premise in a syllogism" (A300). We say that it belongs among axioms - the "universal cognitions a priori" (A300). Since humans "cognize it in pure intuition" (B357), we "would therefore call 'a cognition from principles' that cognition in which I cognize the particular in the universal through concepts" (B357). In addition, "every syllogism is a form of derivation of a cognition from a principle" (B357). Since "a cognition from a principle" (B357) signifies "the category (which constitutes its unity) insofar as it is universal and rests on a rule a priori" (A138/B177-B178), we think that every syllogism, which rests on this cognition, is homogeneous with the category. When humans acquired the function of "a cognition from a principle" (B357) in conjunction with the acquisition of the knowledge in regard to the phenomenon of disappearances among them, death - the pure concept of the understanding - began to serve as the major premise, always giving "a concept such that everything subsumed under its condition can be cognized from it according to a principle" (B357). It can be said that death - the pure concept of the understanding - is the resource, which enables humans to make "synthetic a priori propositions" (B205). Death - nullity in space-time - signifies "an axiom for thinking the totality in the object as real" (A508/B536), opening the way for "the representation I think, which must be able to accompany all others" (B132) to cognize "through categories whatever objects may come before our senses" (B159).

We have to ask again: Why could Kant not attain to the pure concepts of the understanding or synthetic a priori cognition through pure intuition affected by the sensation of nullity - empirical intuition - and synthesis of apprehension? It is because Kant's starting point is too near to modern times in which no attention is paid to "a certain sort of metaphysics" (B21), which "has actually been present in all human beings as soon as reason has extended itself to speculation in them, and it will also always remain there" (B21). Why does Kant have to start from the nearest point? He explains himself, saying, "The series of appearances is to be encountered only in the regressive synthesis itself, but is not encountered in itself in appearance, as a thing on its own given prior to every regress" (A505/B533), implying that Kant himself in virtue of "the representation I think," i.e., "one totality in which all of our representations are contained" (A155) which "is immediately related to the object and is singular" (B377), is not given prior to "the regressive synthesis itself" (A505/B533). Would "the whole series of appearances" (A504/B532) or a "part," which is not given prior to "the regressive synthesis itself" (A505/B533), arise from the mere process of it? No, it cannot. Nevertheless, since Kant believes that "the multiplicity of parts in a given appearance is in itself neither finite nor infinite, because appearance is nothing existing in itself, and the parts are given for the very first time through the regress of the decomposing synthesis" (A505/B533), he absolutely needs to proceed "from the condition proximate to the given appearance toward the more remote conditions" (B438). Kant's "regressive synthesis" (B438) is to be possible in the "dynamical series" (A531/B559), which is supposed to have "the thoroughly conditioned character" (A531/B559). Since Kant has to start from the nearest point in time, he has missed "metaphysics as a natural predisposition" (B22) "metaphysica naturalis" (B21) - which "has actually been present in all human beings as soon as reason has extended itself to speculation in them, and it will also always remain there" (B21). We believe that metaphysica naturalis would be the key to cope with the conundrum in regard to Kant's metaphysics, Hilbert's mathematical problems posed in 1900 (HILBERT 1902: 437-479), and serious defect in quantum mechanics as indicated by Einstein et al. in 1935 (EINSTEIN et al. 1935: 777-780). 
The conundrums in regard to mathematics and quantum mechanics seem to have derived from the same source - Kant's way of thinking in virtue of his metaphysics. Since we believe to have already clarified how to solve the conundrum as regards Kant's metaphysics (YAMAMOTO 2016: 87-100, YAMAMOTO 2017a: 19-37, YAMAMOTO 2017b: 72-81), we launch to make an incipient discourse in an attempt to solve the conundrum as regards to mathematics and quantum mechanics.

\section{Mathematics, Metaphysical Axioms and Synthetic a Priori Proposition}

Kant's metaphysics has a peculiar feature that "the science...namely geometry" (B207) plays a crucial role of determining the direction of the discourse in the Critique of Pure Reason, leading him to make "synthetic propositions" (A33) in terms of the "regulative principle of reason" (A517/B545) and "thing in itself" (A676/B704). Kant himself explains why geometry is so important for his metaphysics, saying, "Appearances are not things in themselves. Empirical intuition is possible only through the pure intuition (of space and time); what geometry says about the latter is therefore undeniably valid of the former, and evasions, as if objects of the senses did not have to be in agreement with the rules of construction in space (e.g., the rules of the infinite divisibility of lines or angles), must cease" (B206); "nothing synthetic could be cognized of them a priori at all, thus not even through pure concepts of space, and the science that they determine, namely geometry, would not itself be possible" (B207). These remarks clearly show that Kant has nothing but geometry in his hands in order to attain to "pure concepts of space" (B207), i.e., space itself. However, Kant's "pure concepts of space" contain the most serious problem, which he will never be able to solve (YAMAMOTO 2016: 87-100, YAMAMOTO 2017a: 19-37, YAMAMOTO 2017b: 72-81). When Kant makes a remark in regard to geometry and mathematics, saying, "The synthesis of spaces and times, as the essential form of all intuition, is that which at the same time makes possible the apprehension of the appearance, thus every outer experience, consequently also all cognition of its object" (B206), we think that Kant falls into the abyss of geometry and mathematics, leading him to think that it might be possible to attain "pure understanding without sensibility" (A242) or "a pure category, in which abstraction is made from any condition of sensible intuition" (B304), through the "logical functions of judgments" (A242). The end product of this "logical functions of judgments" (A242) in conjunction with "all propositions of geometry" (A47) is Kant's "transcendental ideality of appearances" (A506/B534). Here, a serious problem inevitably arises in it. Since "the mathematical axioms (e.g., that there can be only one straight line between any two points)" (A300) are thought to signify "universal cognitions a priori" (A300) in "pure intuition" (B357), Kant's "transcendental ideality of appearances" (A506/B534) - the transcendental ideality of space and time - perennially comprises such things as points, lines and numbers, suggesting that "the mathematical axioms" (A300) are nothing but Kant's "synthetic a priori propositions" (B205). When the transcendental ideality of space and time grounds in the "synthetic a priori propositions" (B205), it is not a universal proposition which "can serve as the major premise in a syllogism" (A300). Why? In Kant's metaphysics, the transcendental ideality of space and time has nothing to do with experience or possible experience since it is merely synthetic a priori. This is absolutely wrong. When "pure category" (B304) is arbitrarily elevated to "principles" (B357), "a cognition from principles" - "that cognition in which I cognize the particular in the universal through concepts" (B357) - becomes possible. Then, "the mathematical axioms (e.g., that there can be only one straight line between any two points)" (A300) - "synthetic a priori propositions" (B205) - emerge in virtue of "pure understanding without sensibility" (A242) or "a pure category, in which abstraction is made from any condition of sensible intuition" (B304). However, actually, Kant himself has divulged that it might be possible to have another mode of transcendental ideality of space and time than he thinks it to be, saying, "I cannot therefore say that in general and in itself I cognize this proposition about straight lines from principles, but only that I cognize it in pure intuition" (A300-B357). Yes, if "pure understanding" (B294) is attained in conjunction with cognizing nullity in space-time through pure intuition affected by the sensation of nullity and synthesis of apprehension, "a pure category, in which abstraction is made from any condition of sensible intuition" (B304) would arise transcendentally through the "logical functions of judgments" (A242). Here "a pure category" is meant to signify death itself - nullity in space-time, i.e., space-time itself.

Therefore, in contrast to Kant's "synthetic a priori propositions" (B205), we make our synthetic a priori propositions, which say that 1) "transcendental ideality of appearances" (A506/B534) - the transcendental ideality of space and time - signifies nullity in space-time, i.e., space-time itself, 2) "the mathematical axioms (e.g., that there can be only one straight line between any two points)" 
(A300) arises not as a principle but as rules for "the analogy by which we utilize concepts of experience in making some sort of concept of intelligible things, with which we have not the least acquaintance as they are in themselves" (A566/B594). We think that the emergence of "transcendental ideality of appearances" (A506/B534) is transcendental, grounded on "possibility itself" (B294) - the possibility of death - while that of "the mathematical axioms" (A300) is rational, grounded on rules or logic. Kant's "mathematical axioms" (A300) - the "logical functions of judgments" (A242) in conjunction with "all propositions of geometry" (A47) - seem to be in contrariety with our synthetic $a$ priori propositions, which states that the transcendental ideality of space and time - nullity in spacetime - belongs to the axioms, i.e., the "universal proposition" (A300). Since "it is taken from experience (by deduction)" (A300), it "can serve as the major premise in a syllogism" (A300). We have already clarified how a universal proposition in regard to space and time - nullity in space-time, i.e., space-time itself - can be drawn from experience or possible experience by deduction (YAMAMOTO 2016: 87-100, YAMAMOTO 2017a: 19-37, YAMAMOTO 2017b: 72-81). Therefore, here we should make a "universal proposition" in virtue of synthetic a priori propositions metaphysical axioms.

Our metaphysical axioms are as follows:

- In experience or in possible experience, sequences of an occurrence in which something happens that previously existed can be ascribed to death itself - object in itself.

- Appearances themselves signify things in themselves, i.e., filled space-elapsing time or empty space-nullified time.

- $\quad$ Space-time itself - nullity in space-time - inheres in filled space-elapsing time.

- Upon disappearance of the manifold of sensible intuition in death, filled space-elapsing time is neutralized, and it would vanish in nullity in space-time, returning to space-time itself.

- Intuitions' condition belongs to one and the same series of intuitions, i.e., space-time itself.

- Space-time itself and the form of appearance in terms of filled-elapsing or empty-nullified correspond to the existence of object at all times.

- A necessary being could exist as filled space-elapsing time, which appears distinct from empty space-nullified time, i.e., nullity in space-time.

- A necessary being - space-time itself - is the unalterable, i.e., the cause.

- All appearances, considered extensively as well as intensively, are continuous magnitudes.

- The absolute whole of magnitude (the world-whole) - the universe - corresponds to appearances themselves.

Here, space-time itself - nullity in space-time - corresponds to the transcendental ideality of space and time. Therefore, we have said, in an opposite manner to what Kant refers to in regard to time (B52-A36), that we do not dispute all claims of time to absolute reality, namely where it would attach to things absolutely as a condition or property, even without regard to the form of sensible intuition. Such properties, which pertain to things in themselves, can be given to us through the senses. In this, therefore, consists the transcendental ideality of time, according to which it is nullity in time, even if one does not abstract from the subjective conditions of sensible intuition, and can be counted as either subsisting or inhering in the objects in themselves (YAMAMOTO 2017a: 19-37). Furthermore, we have said, in an opposite manner to what Kant refers to (A28), that we assert the empirical reality of space (with respect to all possible outer experience), and to be sure its transcendental ideality, i.e., that it is nullity in space if we do not leave aside the condition of the possibility of all appearance, and take it as something that grounds the things in themselves (YAMAMOTO 2017a: 19-37). We have added, saying, in opposition to Kant (A30), that the transcendental concept of appearances in space is a critical reminder that 1) nullity that is intuited in space is a thing in itself: 2) space is a condition that is proper to anything in itself: 3) objects in themselves are known to us; and that 4) what we call outer objects are not mere representations of our sensibility, whose condition is space itself, and whose true correlate, i.e., the thing in itself, is, and can be, cognized through them, and is also sought after in experience or in possible experience (YAMAMOTO 2017a: 19-37). 


\section{Kazuhiko Yamamoto}

It is absolutely universal and necessary for humans to cognize nullity in space-time upon encountering death itself in experience or in possible experience. We have to note that possible experience - the possibility of experience of death - indicates its "possibility itself" (B294), in which its "possibility as a priori cognitions of objects of an intuition in general was exhibited" (B159). On the grounds of " $a$ priori principles of the possibility of experience" (B294) - of nullity in space-time - humans could have the pure concepts of the understanding - death - by cognizing it through pure intuition affected by the sensation of nullity - empirical intuition - and synthesis of apprehension (YAMAMOTO 2016: 87-100, YAMAMOTO 2017a: 19-37). Since nullity in space-time belongs to the "synthetic a priori propositions" (B205), it is not "a singular proposition" (B205) but a "universal proposition" (A300). We have to acknowledge that "it is only the synthesis of that which is homogeneous (of units) that is at issue here" (B205) - synthesis of space-time itself - while "the synthesis here can take place only in a single way" (B205). However, in view of the fact that the subsequent use of the pure concept of the understanding is general among humans, such propositions must therefore be called "axioms" (A165). Since our universal propositions in virtue of axioms signify "universal cognitions a priori" (A300), it "can serve as the major premise in a syllogism" (A300). They are the principles absolute "to the cases that can be subsumed under them" (A300). When Kant says, "I cognize it in pure intuition. I would therefore call a 'cognition from principles' that cognition in which I cognize the particular in the universal through concepts" (B357), we entirely agree with him. Furthermore, we say that our "syllogism is a form of derivation of a cognition from a principle" (B357), since "the major premise always gives a concept such that everything subsumed under its condition can be cognized from it according to a principle" (B357). What is the major premise? It is nullity in space-time - the product of our "metaphysical deduction" in which "the origin of the a priori categories in general was established through their complete coincidence with the universal logical functions of thinking" (B159), namely categorical syllogisms. Since "the categorical syllogisms, whose major premise, as a principle, states the relation of a predicate to a subject" (A406-B433) correspond to general logic which "abstracts from all contents of the predicate" (A72), our metaphysical axioms all converge into nullity in space-time - space-time itself. Seeing that this nullity in space-time or space-time itself is equivalent to "the a priori categories in general" (B159), we say that our metaphysical axioms, homogeneous with categorical syllogisms, are applicable to appearances themselves, indicating that it can go to explain not only "the possibility of things in the world of sense" (A677/B705) but as far as "the possibility of a world-whole itself" (A677/B705) - the universe. Therefore, the "transcendental ideality of appearances" (A506/B534) can be thought to mean that appearances themselves are to exist irrespective of humans in virtue of "things in the world of sense" (A677/B705) or "a world-whole itself" (A677/B705). In other words, it designates that while "appearances in general are nothing outside our representations" (A507), appearances themselves are things to exist outside our representations.

Our metaphysical axioms lead us to make "synthetic a priori propositions" in regard to mathematics, which speak that $i^{2}=-1$ - "imaginary number" in mathematics - would be called "real number" along with 0 and 1 , since $i^{2}=-1,0$ and 1 epitomize reality, which assigns humans to cognize appearances themselves through pure intuition affected by the sensation of nullity - empirical intuition - and synthesis of apprehension. The synthetic a priori propositions preach that 1) real number in mathematics except 0 or 1 should be thought to signify imaginary number, 2) 0 or 1 and so-called "imaginary number" $i^{2}=-1$ should signify a real number (YAMAMOTO 2017b: 72-81). Therefore, we make axiomatic propositions in regard to these numbers that 1 ) the property which belongs to 0 , namely nullity in space-time is followed by the property which belongs to imaginary number $i^{2}=-1$, namely filled space-elapsing time - quantum - 2) when the property which belongs to "imaginary number" $i^{2}=-1$ attains the property which belongs to 1 , it plunges into nullity in space-time, i.e., into the property which belongs to 0 . The synthetic a priori propositions, grounded on metaphysical axioms, seem to be incompatible with those, grounded on mathematical axioms. When Peano axioms (RUSSELL 1920: 1-12) are paraphrased as follows, they seem to exemplify Peano's "synthetic propositions" (A33).

- Axiom One: 0 is a natural number.

- Axiom Two: Every natural number has a successor.

- Axiom Three: 0 is not the successor of any natural number.

- Axiom Four: If the successor of $x$ equals the successor of $y$, then $x$ equals $y$. 
- Axiom Five: If a statement is true of 0 , and if the truth of that statement for a number implies its truth for the successor of that number, then the statement is true for every natural number.

Provided we accept that the "presupposition" (A681/B709) in regard to "the three primitive ideas in Peano's arithmetic...0, number, successor" (RUSSELL 1920: 1-12) signifies the "universal cognitions a priori" (A300), these statements should be regarded as axioms. However, when we examine these axioms, we note that these axioms are not commensurable with metaphysical axioms. Thinking that a "presupposition" cannot be the assertion "of an actual thing to which one would think of ascribing the ground for the systematic constitution of the world" (A681/B709), we cannot erase the doubts that Peano axioms might be "the presupposition itself" (A676/B704) - "reason's speculative interest and not its insight which justifies it in starting from a point lying so far beyond its sphere in order to consider its objects in one complete whole" (A676/B704). The most serious problem resides in Axiom Five. When it says, "If a statement is true of 0 ," we have to know how Peano makes true statements as regards to 0. Furthermore, we have to know how Peano decides the succession of numbers. Only when we hear something true about these things from Peano or someone else, we will accept Peano axioms. If we try to dovetail Peano axioms for natural numbers with metaphysical axioms for our real number: $0, i^{2}=-1,1$, the axioms are to be as follows.

- Axiom One: 0 is a real number.

- Axiom Two: Every real number has a successor.

- Axiom Three: 0 can be the successor of any real number.

- Axiom Four: Even if the successor of $x$ equals the successor of $y, x$ does not necessarily equal $y$.

- Axiom Five: If a statement is true of 0 , and if the truth of that statement for a number implies its truth for the successor of that number, then the statement is true for every real number.

We think that Peano axioms, if Axiom Three and Axiom Four are rephrased, are commensurate with metaphysical axioms, which can determine something true concerning "the three primitive ideas in Peano's arithmetic...0, number, successor" (RUSSELL 1920: 1-12). These axioms, in commensurate with metaphysical axioms, indicate that 1) 0 is to signify nullity in space-time - space-time itself while number to signify infinite steps of alteration of filled space-elapsing time or its consummation or nullity in space-time; 2) succession is to signify the alteration from nullity in space-time to a part of filled space-elapsing time or from a part of filled space-elapsing time to empty space-nullified time, i.e., nullity in space-time, suggesting that 1$) i^{2}=-1$ is to signify infinite steps of alteration of spacetime itself - quantum - between filled-elapsing and empty-nullified, 2) $0=$ nullity in space-time space-time itself - is to permeate filled space-elapsing time, 3) $i^{2}=-1$ is to come across its cessation any time before the consummation of itself as number 1 .

Furthermore, we can deduce from the Peano-metaphysical axioms more "universal propositions" as follows:

1) All alteration as a transition of a thing from one state to another signifies: 1) an alteration of a part of filled space-elapsing time to empty space-nullified time: 2) a passing out of a part of filled space-elapsing time into a part of filled space-elapsing time as the succession of the states itself: 3) an alteration of empty space-nullified time to a part of filled space-elapsing time.

2) It is impossible for a part of filled space-elapsing time and another part of filled space-elapsing time to be at the same point in the same instance.

3) It is possible for a part of empty space-nullified time and another part of empty space-nullified time to be at the same point in the same instance - nullity in space-time - if points and instances are conjured up in nullity in space-time.

4) The form of appearance, which alterability concerns, is filled-elapsing or empty-nullified, while their cause is in the unalterable - space-time itself.

5) All appearances arise in a spontaneity, which could start to act from itself, without needing to be preceded by any other cause that in turn determines it to action according to the law of causal connection.

6) The synthesis of the manifold part of space-time itself is successive, and thus contains a series. 
7) The synthesis of the manifold part of space-time itself takes place in the manifold of sensibility filled space-elapsing time.

8) Succession, subordination and coordination which take place in filled space-elapsing time affect the world-whole.

It is clear that the "Common Notions" (EUCLID 2002:1-36) in Euclid's Elements are also incompatible with mathematical axioms.

Common Notions speak:

1. Things which are equal to the same thing are also equal to one another.

2. If equals be added to equals, the wholes are equal.

3. If equals be subtracted from equals, the remainders are equal.

4. Things which coincide with one another are equal to one another.

5. The whole is greater than the part.

Metaphysical axioms ordains: 1) Since there is nullity in space-time which is equal to nullity in spacetime in the world-whole, a thing - nullity in space-time - which is equal to the same thing, is equal to one another. On the contrary, since there is no part of filled space-elapsing time which is equal to the same part of filled space-elapsing time in the world-whole, things - parts of filled space-elapsing time - which are not equal to the same thing, are not equal to one another: 2) since there is nullity in spacetime which is equal to nullity in space-time in the world-whole, if equal - nullity in space-time - is added to equal, i.e., nullity in space-time, the wholes are equal. On the contrary, since there is no part of filled space-elapsing time which is equal to another part of filled space-elapsing time in the worldwhole, it is impossible for equal to be added to equal: 3 ) since there is nullity in space-time which is equal to nullity in space-time in the world-whole, if equal - nullity in space-time - is subtracted from equal, i.e., nullity in space-time, the remainders are equal. On the contrary, since there is no part of filled space-elapsing time which is equal to another part of filled space-elapsing time in the worldwhole, it is impossible for equal to be subtracted from equal: 4) since there is nullity in space-time which coincides with one another in the world-whole, things - nullity in space-time - are equal to one another. On the contrary, since there are no parts of filled space-elapsing time which coincide with one another, things - parts of filled space-elapsing time - are not equal one another: 5) since the whole consists of filled space-elapsing time and nullity in space-time, and nullity in space-time permeates filled space-elapsing time, the whole - nullity in space-time - cannot be greater than the part - nullity in space-time.

Thus, Common Notions are to be neutralized by metaphysical axioms. Why does the contrariety between mathematical axioms and metaphysical axioms occur? Kant again gives us a clue to the answer, saying "one necessarily thinks of the fully elapsed time up to the present moment as also given (even if not as determinable by us). But as to the future, since it is not a condition for attaining to the present, it is a matter of complete indifference for comprehending the present what we want to hold about future time, whether it stops somewhere or runs on to infinity" (B437). We think that there are serious defects in this way of thinking. First, "the fully elapsed time up to the present moment" (B437) cannot be thought as given, since elapsed time - nullity in time - is nothing but a condition while only elapsing time is given. Second, since the future - nullity in time - is "a condition for attaining to the present" (B347), it is a matter of complete interest "for comprehending the present what we want to hold about future time, whether it stops somewhere or runs on to infinity" (B437). Therefore, on account of the fact that Peano axioms and Common Notions ground in the abstraction that can "occur without the restrictions that nature has attached to them" (B57), i.e., the abstraction "from everything empirical in the appearances" (A96), we say that Peano axioms and Common Notions, which are "first abstracting a predicate of a thing from its concept and subsequently connecting its opposite with this predicate, which never yields a contradiction with the subject, but only with the predicate that is combined with it synthetically" (A153), have been used as "the mathematical principles, which are constitutive" (B223). It is clear that the product of "first abstracting a predicate of a thing from its concept" (A153) is nothing but nullity, while "its opposite with this predicate" (A153) signifies "all propositions of geometry" (A47). Since connecting nullity with "all propositions of geometry" yields no contradiction with the subject, the predicate can be 
freely combined with the subject synthetically. However, when nullity and "all propositions of geometry" (A47) are affirmed at the same time, namely "when both the first and the second predicate are affirmed at the same time" (A153), something like this could occur: "with two straight lines no space at all can be enclosed, thus no figure is possible, and try to derive it from the concept of straight lines and the number two;..." (B65). We have to say that since Peano axioms and Common Notions could comprise this intractable problem, they are not to pertain to appearance itself but to analogy or mere appearance. On the contrary, metaphysical axioms can be said to predict that "the a priori concepts of space and time" (B57) are not "creatures of the imagination, the origin of which must really be sought in experience, out of whose abstracted relations imagination has made something that, to be sure, contains what is general in them, but that cannot occur without the restrictions that nature has attached to them" (B57). On account of the fact that "the a priori concepts of space and time" (B57) - space-time itself - are not creatures of the imagination, the so-called imaginary number $i^{2}=-1$ is regarded to be real number, which is to signify "one reality, if combined in one subject with another, cancels out the effect of the latter, which is unceasingly placed before our eyes by all hindrances and counter-effects in nature, which, since they rest on forces, must be called realitates phaenomena" (B329). On the ground of this discourse, we say that mathematics and geometry signify "a merely formal (a priori) consciousness of the manifold in space and time" (B208). However, "a merely formal (a priori) consciousness" (B208), as "unbounded reality" (B322), is to comprehend magnitude through the "synthesis of the generation of the magnitude of a sensation from its beginning, the pure intuition $=0$, to any arbitrary magnitude" (B208). Peano axioms and Common Notions can be thought to pertain to the analogy of "the real, which corresponds to sensations in general, in opposition to the negation $=0 "$ " (B217), which arises when space itself is filled and time itself is elapsing (YAMAMOTO 2017a: 19-37) - quantum discretum - while forgetting "quantum continuum" (A527/B555). Therefore, if "a merely formal (a priori) consciousness" (B208) mathematics or geometry - is enhanced with "the matter of all possibility" (B322) by means of metaphysical axioms, it would become possible, through mathematics or geometry, "to anticipate experience precisely in what concerns its matter, which one can draw out of it" (B209) in analogy. Then, how to enhance mathematics or geometry with metaphysical axioms?

\section{Intuition, Continuity, and Mathematical Problems}

In regard to the issue of relations between mathematics, logic and intuition, Klein had a penetrating view, which says, "I have to point out most emphatically...that it is not possible to treat mathematics exhaustively by the method of logical deduction alone, but that, even at the present time, intuition has its special province" (KLEIN 1896: 241-249). In addition, he says, "I do not grant that the arithmetized science is the essence of mathematics," since it "began originally, as I pointed out, by ousting space intuition" (KLEIN 1896: 241-249). On account of the fact that "G. Canto has opened up new spheres of thought to arithmetical science," picturing "before us in space an infinite number of points and forms composed of them," the issue of "the totality of points in space as a numbermanifoldness in which we interpolate the irrational numbers in the usual manner between the rational numbers arranged in three dimensions" (KLEIN 1896: 241-249) comes forwards. In order for the arithmetized science to attain to the position of essence of mathematics, it has to find a solution to the issue of continuity of space on the arithmetical foundation of analysis. Klein, asserting that mere mathematics cannot do it with the use of the "cultivated intuition" such as a "mathematical intuition," makes it clear that it needs rather "the naïve intuition, largely a natural gift" (KLEIN 1896: 241-249). Actually, "in the development of most of the branches of our science, intuition was the stating point, while the logical treatment followed" (KLEIN 1896: 241-249). Therefore, when "we ultimately perceive that space intuition is an inexact conception" in terms of nullity in space-time - the pure concept of the understanding - we "idealize it by means of the so-called axioms, which actually serve as postulates" (KLEIN 1896: 241-249), namely metaphysical axioms. Since metaphysical axioms rest on "proofs from experience" (A85), its "pure use a priori (completely independently of all experience)" (A85), which requires "a deduction of their entitlement" (A85), is always lawful. Since our transcendental deduction of objects a priori rests on "the empirical deduction, which shows how a concept is acquired through experience and reflection on it" (A85), it can be said that metaphysical axioms - the metaphysical deduction - has enhanced lawfulness. When the "intuition has completed the task of idealization" (KLEIN 1896: 241-249), logical investigation is in place now. Metaphysical axioms would logically lead us to another axiom, which says that continuity of space cannot be 
attained without the arithmetical foundation of analysis in virtue of so-called "imaginary number" $i^{2}=$ -1 along with real numbers 0 and 1 . The $i^{2}=-1$ is supposed to be a "real number," which pertains to "that magnitude which can only be apprehended as a unity, and in which multiplicity can only be represented through approximation to negation $=0$, intensive magnitude" (B210). Since "every reality in the appearance has intensive magnitude, i.e., a degree" (B210), "this reality as cause (whether of the sensation or of another reality in appearance, e.g., an alteration)" can be called "the moment of gravity" (B210). "Because, indeed, the degree designates only that magnitude the apprehension of which is not successive but instantaneous" (B210-A169), it grounds in "pure a priori imagination" (A142) or "a pure a priori intuition" (A48) - the pure concepts of the understanding. Thus, the $i^{2}=-1$ is commensurate with "one that is also merely empirical, i.e., a proposition of experience" (A47), which can "contain necessity and absolute universality" (A47). According to Klein, "Mathematical developments originating in intuition must be not considered actual constituents of the science till they have been brought to a strictly logical form" (KLEIN 1896: 241-249). Here, we say that our metaphysical axioms are to be considered actual constituents of mathematics since they have been brought to a strictly logical form. Furthermore, we say, in contrast to Klein, that the number $i^{2}=-1$ must be interpolated in the usual manner between real numbers 0 and 1 arranged in four dimensions, which, we suppose, can be a clue to the solution in regard to the issue of continuity of space and time on the arithmetical foundation of analysis.

In regard to this issue, Hilbert made a discourse in his well-known lecture delivered at the International Congress of Mathematics in Paris in 1900. Among the twenty-three mathematical problems Hilbert raised in this lecture, Cantor's problem of the cardinal number of the continuum was the first that was expected to be solved in the $20^{\text {th }}$ century (HILBERT 1902: 437-479). Here, Hilbert first defines Cantor's problem as follows: "Two systems, i.e., two assemblages of ordinary real number or points, are said to be (according to Cantor) equivalent or of equal cardinal number, if they can be brought into a relation to one another such that to every number of the one assemblage corresponds one and only one definite number of the other" (HILBERT 1902: 437-479). Even if Cantor asserts that "Every system of infinitely many real number, i.e., every assemblage of numbers

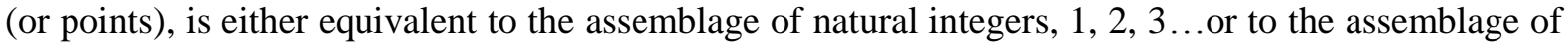
all real numbers and therefore to the continuum, that is, to the points of a line; as regards equivalence there are, therefore, only two assemblages of numbers, the countable assemblage and continuum" (HILBERT 1902: 437-479), the most serious problem arises in terms of the theorem that "the continuum has the next cardinal number beyond that of the countable assemblage" (HILBERT 1902: 437-479). In other words, the issue of what the cardinal number signifies inevitably comes forwards. In order to clarify it, "a new bridge between the countable assemblage and the continuum" (HILBERT 1902: 437-479) is absolutely necessary. However, it would never come out from mathematical axioms. From where would it come out? It would come out only through metaphysical axioms. Kant elaborates on it as follows: "since every reality has its degree that can decrease to nothing (emptiness) through infinite steps while the extensive magnitude of the appearance remains unaltered, it must yield infinitely different degrees with which space or time is filled, and the intensive magnitude in different appearances can be smaller or greater even though the extensive magnitude of the intuition remains identical" (B214-A173). We think that what Kant refers to here indicates that $0, i^{2}=-1$, and 1 would signify cardinality. Since the numbers $0, i^{2}=-1$, and 1 can be regarded as a "real number" (YAMAMOTO 2017b: 72-81), metaphysical axioms dictate that; 1) 0 signifies nullity in space-time, 2) number 1 signifies the consummation of filled space-elapsing time, and 3) $i^{2}=-1$ signifies every reality, which "has its degree that can decrease to nothing (emptiness) through infinite steps" (B214A173). We have to think that $i^{2}=-1$ is the bridge between the countable assemblage and continuum. Then, a question of how this "system of real numbers is said to be ordered" (HILBERT 1902: 437479) comes forward, since "for every two numbers of the system it is determined which one is the earlier and which the later, and...at the same time this determination is of such a kind that, if $a$ is before $b$ and $b$ is before $c$, then $a$ always comes before $c$ " (HILBERT 1902: 437-479). If "the natural arrangement of numbers of a system is defined to be that in which the smaller precedes the larger" (HILBERT 1902: 437-479), why is it? The answer cannot be deduced from mathematical axioms but only from metaphysical axioms. Since the causality does not reside in numbers themselves but in the universe, we must seek it in metaphysica naturalis. We have already clarified that 1) the form of the universe is filled-elapsing or empty-nullified, while their cause is space-time itself, 2) the universe arises in a spontaneity, which could start to act from itself, without needing to be preceded by any other cause that in turn determines it to action according to the law of causal connection, 3) 
succession, subordination and coordination which take place in filled space-elapsing time could affect the universe. Since there is no first thing in the universe, "not only in the assemblage itself but also in every partial assemblage there exists" (HILBERT 1902: 437-479) no first number. However, since succession, subordination and coordination take place in filled space-elapsing time, it is clear that "for every two numbers of the system it is determined which one is the earlier and which the later and...at the same time this determination is of such a kind that, if $a$ is before $b$ and $b$ is before $c$, then $a$ always comes before $c$ " (HILBERT 1902: 437-479). Therefore, we say that $i^{2}=-1$ is before 1 , and 0 is before $i^{2}=-1$, then 0 always comes before 1 . Furthermore, we have to say that 1 always comes before 0 . The cardinals: $0, i^{2}=-1$, and 1 are thought to signify the "assemblages of ordinary real numbers or points" (HILBERT 1902: 437-479), while so-called "real number" in mathematics except 0 or 1 is the partial system, which "will also prove to be ordered" (HILBERT 1902: 437-479). Metaphysical axioms make it clear that Hilbert's thought that "the system of all real numbers, i.e., the continuum in its natural order, is evidently not well ordered" (HILBERT 1902: 437-479) is a mere presupposition with little foundation. The mathematics cannot answer the question of "whether the totality of all numbers may not be arranged in another manner so that every partial assemblage may have a first element" (HILBERT 1902: 437-479). The only way to "obtain a direct proof of this remarkable statement of Cantor's, perhaps by actually giving an arrangement of numbers such that in every partial system a first number can be pointed out" (HILBERT 1902: 437-479) is through the transcendental analytic and metaphysical axioms, which rest on the metaphysics in the Critique of Pure Reason (YAMAMOTO 2016: 87-100, YAMAMOTO 2017a: 19-37, YAMAMOTO 2017b: 72-81). Metaphysical axioms dictates that since the system of all real numbers, i.e., the continuum in its natural order, is evidently well ordered, in every partial system a first number can be pointed out.

In regard to the issue of why mathematics cannot solve the fundamental problem, Kant makes a penetrating discourse, saying "Nearly all natural philosophers, since they perceive a great difference in the quantity of matter of different sorts in the same volumes (partly through the moment of gravity, or weight, partly through the moment of resistance against other, moved matter), unanimously infer from this that this volume (extensive magnitude of the appearance) must be empty in all matter, although to be sure in different amounts. But who among these for the most part mathematical and mechanical students of nature ever realized that their inference rested solely on a metaphysical presupposition, which they make so much pretense of avoiding? - for they assume that the real in space...is everywhere one and the same, and can be differentiated only according to its extensive magnitude, i.e., amount" (B215). We think that since "for the most part mathematical and mechanical students of nature" (B215) have inferred that "this volume (extensive magnitude of the appearance) must be empty in all matter, although to be sure in different amounts" (B215), following "nearly all natural philosophers" (B215) who have unanimously inferred this from the perception of "a great difference in the quantity of matter of different sorts in the same volumes" (B215), the fundamental problem of mathematics could not have happened. However, actually, it has happened. We must know why it has happened. According to Kant, it has happened because of the metaphysical presupposition that "the real in space...is everywhere one and the same, and can be differentiated only according to its extensive magnitude, i.e., amount" (B215). Whose presupposition is it? It is Kant's metaphysical presupposition. On the contrary, we have to think that the real in space itself...is everywhere one and the same, and can be differentiated only according to its intensive magnitude, i.e., "the quantity of matter of different sorts in the same volumes (partly through the moment of gravity, or weight, partly through the moment of resistance against other, moved matter)" (B215). We would say, in an opposite manner to what Kant refers to (B215-A174-B216), that this is "all possible laws according to which the elements of a fundamental sequence may proceed" (HILBERT 1902: 437-479), for which we can have ground in experience or in possible experience and which is therefore empirical and metaphysical. We present an axiomatic proof which, to be sure, will explain the variation in the filling of space, and which will entirely obviate the alleged necessity of the presupposition that the difference in question cannot be explained by the assumption of empty space...Furthermore, It will explain that everything real has for the same quality its degree (of resistance or of weight) which, without diminution of the extensive magnitude or amount, can become infinitely smaller until it is transformed into emptiness and disappears. Our cardinals $0, i^{2}=-1$, and 1 are supposed to signify this "everything real" which "has for the same quality its degree (of resistance or of weight) which, without diminution of the extensive magnitude or amount, can become infinitely smaller" (B216). When Hilbert utters a negative remark in regard to $i^{2}=-1$, saying "If contradictory attributes be assigned to a concept, I say, 
that mathematically the concepts does not exists. So, for example, a real number whose square is -1 does not exist mathematically" (HILBERT 1902: 437-479), we have to say, on the contrary, that since $i^{2}=-1$ signifies reality (YAMAMOTO 2017b: 72-81), a real number whose square is -1 does exist mathematically on the grounds of metaphysical axioms. Since "the proof for the compatibility of the axioms shall be fully accomplished," we think that what Hilbert says is to be corroborated, which says, "the proof of the compatibility of the axioms is at the same time the proof of the mathematical existence of the complete system of real numbers or of the continuum" (HILBERT 1902: 437-479). Even if the abstraction can "occur without the restrictions that nature has attached to them" (B57), mathematical axioms must rest on the abstraction which cannot occur without the restrictions that nature has attached to them. In this regard, we say that the axiom in regard to $i^{2}=-1$, which rests on metaphysical axioms, belongs among mathematical axioms.

\section{Geometry, Numbers, and Metaphysical Axioms}

In regard to the geometrical axioms, Hilbert has made the most important and penetrating discourse, saying, "In geometry, the proof of the compatibility of the axioms can be effected by constructing a suitable field of numbers, such that analogous relations between the numbers of this field correspond to the geometrical axioms. Any contradiction in the deductions from the geometrical axioms must thereupon be recognizable in the arithmetic of this field of numbers" (HILBERT 1902: 437-479). Yes, we have recognized the "contradiction in the deductions from the geometrical axioms" in the arithmetic of this field of numbers as follows: In "all propositions of geometry" (A47), something like this could occur: "with two straight lines no space at all can be enclosed, thus no figure is possible, and try to derive it from the concept of straight lines and the number two;..." (B65). On the contrary, we have already said that in our proposition of experience, an a priori intuition or pure intuition could occur with no straight lines and no number two or three. Space can be enclosed, and thus, the figure is possible here as a "synthetic proposition" (A47), which is "one that is also merely empirical, i.e., a proposition of experience" (A47). We think that, here, if nullity in space-time is introduced in accordance with an a priori intuition or pure intuition, it might neutralize the conundrum, which unavoidably occurs in "all propositions of geometry" (A47). It means that space can be enclosed with two straight lines and the figure is possible, if "figures in space" (A142) are under the aegis of pure $a$ priori imagination (YAMAMOTO 2017b: 72-81). It is through the way of nullity in space-time space-time itself - that "the desired proof for the compatibility of the geometrical axioms is made to depend upon the theorem of the compatibility of the arithmetical axioms" (HILBERT 1902: 437-479).

If "the totality of real numbers, i.e., the continuum...is not the totality of all possible series in decimal fractions, or of all possible laws according to which the elements of a fundamental sequence may proceed" (HILBERT 1902: 437-479), the compatibility of the geometrical axioms with "a suitable field of numbers" (HILBERT 1902: 437-479) would crumble to dust. In regard to this issue, Hilbert says, "It is rather a system of things whose mutual relations are governed by the axioms set up and for which all propositions, and only those, are true which can be derived from the axioms by a finite number of logical process" (HILBERT 1902: 437-479). When Hilbert asks "If, in an isosceles triangle, the ratio of the base angle to the angle at the vertex be algebraic but not rational, the ratio between base and side is always transcendental" (HILBERT 1902: 437-479), suggesting that irrational numbers could be homogenous with being transcendental, we agree with him. Then, it would become possible for us to say "the concept of the continuum or even that of the system of all functions exists" (HILBERT 1902: 437-479) in exactly the same sense "as Cantor's higher classes of numbers and cardinal numbers" (HILBERT 1902: 437-479). We think that our system of metaphysics, which "corresponds best also to what experience and intuition tell us" (HILBERT 1902: 437-479), is correspondent to the "axioms by a finite number of logical processes," which corroborates that "the concept of the continuum or even that of the system of all functions exists" (HILBERT 1902: 437479) in the same sense "as Cantor's higher classes of numbers and cardinal numbers" (HILBERT 1902: 437-479). There, "the system of all cardinal numbers" (HILBERT 1902: 437-479) can be set up on the grounds of metaphysical axioms, which rest on "what experience and intuition tell us" (HILBERT 1902: 437-479), while the system "of all Cantor's alephs" (HILBERT 1902: 437-479) cannot be set up, as indicated above. We think that the former is metaphysically and mathematically existent, while the latter is metaphysically non-existent. Furthermore, in association with the issue of "in isosceles triangle, the ratio of the base angle to the angle at the vertex" (HILBERT 1902: 437479), when Hilbert says that "The expression $\alpha^{\beta}$, for an algebraic base $\alpha$ and an irrational algebraic 
exponent $\beta$, e.g., the number $2^{\sqrt{2}}$ or $e^{\pi}=i^{-2 i}$, always represent a transcendental or at least irrational number" (HILBERT 1902: 437-479), we agree with him, thinking that the expression $\alpha^{\beta}$, for an algebraic base $\alpha$ and a transcendental, irrational algebraic exponent $\beta$, e.g., the number $2^{\sqrt{2}}$ or $e^{\pi}=i^{-2 i}$, represents a transcendental, irrational number - real number. If we make the alteration in the way of logical thinking, the problem that "certain special transcendental functions, important in analysis, take algebraic values for certain algebraic arguments" (HILBERT 1902: 437-479) would not occur. We would say, in an opposite manner to what Hilbert refers to (HILBERT 1902: 437-479), that we do not expect transcendental functions to assume transcendental values for algebraic arguments: and, since it is well known that there exist integral transcendental functions which even have rational values for all algebraic arguments, we consider it highly improbable that the exponential function $e^{i \pi z}$, for example, which has algebraic values for all rational arguments $z$, will on the other hand always take transcendental values for irrational algebraic values of the argument $z$.

Why has this kind of thing: "we expect transcendental functions to assume, in general, transcendental values for even algebraic arguments" (HILBERT 1902: 437-479), happened in mathematics? Did it happen only in mathematics? No. It has happened in all sciences, including philosophy, mathematics and physics. Kant expounds why this has happened in his metaphysics, saying, "That nature should direct itself according to our subjective ground of apperception, indeed in regard to its lawfulness even depend on this, may well sound quite contradictory and strange. But if one considers that this nature is nothing in itself but a sum of appearances, hence not a thing in itself but merely a multitude of representations of the mind, then one will not be astonished to see that unity on account of which alone it can be called object of all possible experience, i.e., nature, solely in the radical faculty of all our cognition, namely transcendental apperception; and for that very reason we can cognize this unity a priori, hence also as necessary, which we would certainly have to abandon if it were given in itself independently of the primary sources of our thinking. For then I would not know whence we should obtain the synthetic propositions of such a universal unity of nature, since in this case one would have to borrow them from the objects of nature itself. But since this could happen only empirically, from that nothing but merely contingent unity could be drawn..." (A114). As we have already clarified, this way of thinking: "transcendental apperception" signifies "merely contingent unity" is absolutely wrong (YAMAMOTO 2016: 87-100, YAMAMOTO 2017a: 19-37, YAMAMOTO 2017b: 72-81). Because of this way of thinking, Kant has to think up his "thing in itself" (A676/B704), which is an epistemological naught. In contrast to this, we have shown, by means of the "categorical syllogism" (A406) in conjunction with pure intuition affected by the sensation of nullity - empirical intuition and synthesis of apprehension, that "a thing in itself" is to signify nullity in space-time, i.e., spacetime itself (YAMAMOTO 2016: 87-100, YAMAMOTO 2017a: 19-37). Therefore, when Kant raises questions, saying, "But that empirical rule of association, which one must assume throughout if one says that everything in the series of occurrences stands under rules according to which nothing happens that is not preceded by something upon which it always follows - on what, I ask, does this, as a law of nature, rest, and how is this association even possible? The ground of the possibility of the association of the manifold, insofar as it lies in the object, is called the affinity of the manifold. I ask, therefore, how do you make the thoroughgoing affinity of the appearances (by means of which they stand under constant laws and must belong under them) comprehensible to yourself?" (A112-A113), we have answered these questions. Our transcendental analytic have clarified that 1) "empirical rule of association" is meant to signify death itself, 2) the "rule of association," under which everything in the series of occurrences stands, is possible through "occurrences" - succession, subordination and coordination of categories - 3) "the affinity of the manifold" is meant to signify space-time itself, 4) "the thoroughgoing affinity of the appearances" is meant to signify nullity in space-time - space-time itself (YAMAMOTO 2016: 87-100, YAMAMOTO 2017a: 19-37, YAMAMOTO 2017b: 72-81). Thus, we believe that we have solved a conundrum in regard to philosophy. How about mathematics? Yes, we feel that we have a clue to solve the conundrum in regard to mathematics, as indicated above. How about physics? Probably we have obtained a clue to solve the conundrum in regard to physics as well. We say, in an opposite manner to what Kant refers to (B213), that since we have before us here everything that we can use - the pure fundamental concepts of all possible experience, in which there must be everything empirical - we can anticipate general natural science, which is built upon certain fundamental experiences, without injuring the unity of the system. 


\section{REFERENCES}

[1] Einstein, Albert, Podolsky, Boris, Rosen, Nathan, "Can Quantum-Mechanical Description of Physical Reality Be Considered Complete?," Physical Review 47: 777-780, 1935.

[2] Euclid, Euclid's Elements, Green Lion Press, 2002.

[3] Hilbert, David, "Mathematical Problems," Bulletin of the American Mathematical Society 8: 437-479, 1902.

[4] Kant, Immanuel, Critique of Pure Reason, Cambridge University Press, 1999.

[5] Klein, Felix, "The Arithmetizing of Mathematics," Bulletin of the American Mathematical Society 2: 241-249, 1896.

[6] Russell, Bertrand, Introduction to Mathematical Philosophy, The Macmillan, 1920.

[7] Yamamoto, Kazuhiko, "The Pure Concepts of the Understanding and Synthetic A Priori Cognition: the Problem of Metaphysics in the Critique of Pure Reason and a Solution," Proceedings of the European Conference on Ethics, Religion \& Philosophy 2016, pp 87-100, 2016.

[8] Yamamoto, Kazuhiko, "The Transcendental Aesthetic and Absolute Totality of Conditions: The Problem of Metaphysics in the Critique of Pure Reason and a Solution," International Journal of Humanities Social Sciences and Education 4 (2): 19-37, 2017 a.

[9] Yamamoto, Kazuhiko, "Absolute Totality, Causality, and Quantum: The Problem of Metaphysics in the Critique of Pure Reason," International Journal of Humanities Social Sciences and Education 4 (4): 72-81, $2017 \mathrm{~b}$. 